\title{
AUTOMATIC EXTRACTION OF WATER BODIES FROM TERRASAR-X DATA
}

\author{
Thomas Hahmann, Achim Roth, Sandro Martinis, André Twele, Astrid Gruber \\ German Aerospace Center (DLR) \\ German Remote Sensing Data Center \\ Oberpfaffenhofen, 82234 Wessling, Germany \\ (thomas.hahmann, achim.roth, sandro.martinis, andre.twele, astrid.gruber)@dlr.de
}

\begin{abstract}
Medium resolution SAR satellite data have been widely used for water and flood mapping in recent years. Since the beginning of 2008 high resolution radar data with up to one meter pixel spacing of the TerraSAR-X satellite are operationally available. The improved ground resolution of the system offers a high potential for water detection. However, image analysis gets more challenging due to the large amount of image objects that are visible in the data. Water body detection methods are reviewed with regard to their applicability for TerraSAR-X data. Flood detection approaches for rapid disaster mapping are presented in this paper.
\end{abstract}

Index Terms - Synthetic aperture radar, TerraSAR-X, Water body detection, flood mapping, image analysis

\section{INTRODUCTION}

Due to their cloud penetration capability, SAR satellites are almost independent from weather and daylight. Therefore they are more suitable than optical sensors to reliably and timely map inundated areas in flood situations, which usually occur under overcast sky conditions.

Since nearly two decades operational space borne systems like ERS-1/2, Envisat ASAR and Radarsat-1 have been used to map water bodies and flood situations at CBand wavelength independent of cloud coverage with medium resolution. The ALOS PALSAR sensor provides the possibility to study water features at L-Band wavelength since its launch in 2006.

Recent development shows the advent of several high resolution SAR satellites. At June 15, 2007 the German XBand TerraSAR-X satellite was launched. With the end of the commissioning phase operational data acquisition started in January 2008. In this paper, we present first experiences in the application of high-resolution TerraSAR$\mathrm{X}$ data for water body extraction with a focus on flood extent mapping. In 2007 two of four planned COSMOSkyMed satellites (X-Band) and the Radarsat-2 satellite (C-
Band) were successfully launched as well. This fleet of high resolution SAR satellites complementary enables a rapid and timely dense observation capability in the context of crisis response.

Main orbit characteristics of TerraSAR-X are sunsynchronous, near-polar dusk-dawn at a flying altitude of $514 \mathrm{~km}$. The satellite features a nominal repetition rate of 11 days. Each area of interest however can be imaged within two to four days depending on its latitude using a variety of different incidence angles. The versatile antenna of TerraSAR-X has the following imaging capabilities: In the SpotLight (SL) and High Resolution SpotLight (HS) modes, a spatial resolution of up to 1 meter can be achieved. The size of the ground track is either 5 (HS) or 10 (SL) km in azimuth and $10 \mathrm{~km}$ in range. In the StripMap (SM) mode, a scene has a swath width of $30 \mathrm{~km}$ and a maximum length of $1500 \mathrm{~km}$. Depending on the incidence angle, the ground resolution can be up to 3 meters. In the ScanSAR mode (SC) images with a swath width of 100 kilometers and a maximum length of $1500 \mathrm{~km}$ can be acquired at a spatial resolution of 16 meters. For each imaging mode, a variety of different acquisition parameters can be defined (e.g. incidence angle, polarization, processing parameters).

The high resolution and the increased observation frequency of the new class of SAR sensors offer enormous potential in the domain of flood mapping. However, the improved spatial resolution of the SAR data results in a large variety of very small-scaled image objects, which makes image processing and analysis even more challenging. The general aim of the ongoing research activities is the development of pixel-based and objectbased algorithms, which allow near-realtime SAR data processing and reliable water body and flood mapping, especially in complex scenarios such as urban areas.

This article is organized as follows: In section 2 different forms of appearance of water bodies in TerraSAR-X data are presented. Chapter 3 contains a review of different methods for flood extraction and their adaptability to high resolution SAR data. The water body detection concept for the TanDEM-X mission is introduced in section 3.2. Flood extraction approaches in the context of near-real time disaster mapping are described in chapter 3.3. 


\section{DIFFERENT FORMS OF APPEARANCE OF WATER BODIES IN TERRASAR-X DATA}

The availability of high resolution SAR data like from the TerraSAR-X satellite facilitates the image interpreter to distinguish more feature details on the Earth's surface. Water bodies which appear dark in medium resolution SAR imagery like ERS-1/2 or Envisat ASAR may exhibit bright structures in high resolution data. Various different forms of appearance of water bodies can be observed in TerraSAR-X data.

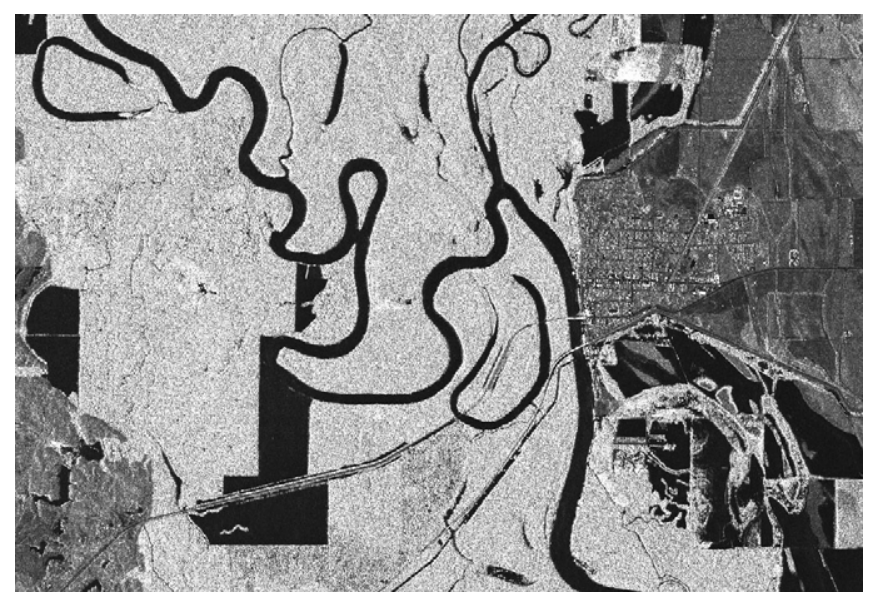

Figure 1. Underflooded forests along the White River at Clarendon, Arkansas (USA), TerraSAR-X StripMap data; ㄷ DLR (2008)

Vegetation within water may increase the backscatter values of the water surface due to double bouncing leading to brighter regions on the water body. The TerraSAR-X scene of the flooded White River valley in figure 1 features open water (dark areas) and underflooded forest areas (very bright regions). The use of dual polarization data can help to distinguish between dry surfaces, vegetation that pokes out of the water and water bodies.

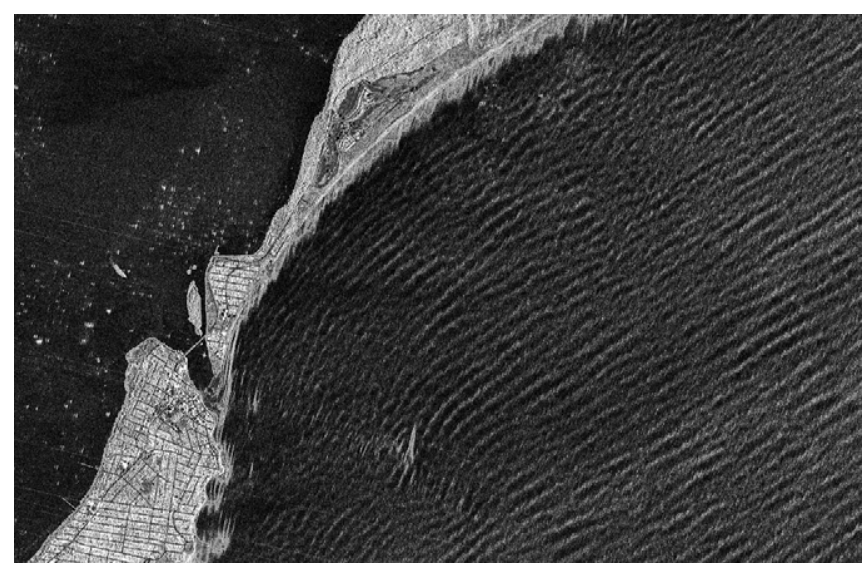

Figure 2. Wave pattern along the Australian Pacific coast near Sydney, TerraSAR-X StripMap data; (C DLR (2008)
Figure 2 shows wave patterns along the Australian coast. The wind speed determines the wave height and as a result the backscatter intensity. The left part of the image shows a shallow lake which has a smooth surface without visible waves. Hence these areas appear darker in the image. Even waves with a wavelength in the range of the sensor resolution of TerraSAR-X of up to $1 \mathrm{~m}$ may be visible as bright linear features on the surface of lakes or the sea. Texture analysis may be useful to use these regular features to correctly detect water bodies.

Rivers are not very susceptible to wind induced waves due to their small width and hence appear predominantly dark in SAR data. If the flow velocity increases the river surface roughens, which can cause increased backscatter values.

Other areas of interest for water mapping are urban areas. Especially in a flood situation the improved pixel spacing of the high resolution TerraSAR-X data supports mapping inundated urban areas more reliably. Auxiliary data like three-dimensional city models are necessary to distinguish between radar shadow areas and water bodies, which both appear dark. A similar problem of discriminating dark water surfaces and adjacent dark radar shadow areas may occur in high mountainous terrain with steep slopes when the SAR data are acquired at shallow incidence angles.

\section{DETECTION OF WATER BODIES IN HIGH RESOLUTION SAR DATA}

\subsection{Comparison of pixel-based and object-based approaches}

For the extraction of water bodies in SAR imagery pixeland segmentation-based classification techniques can be distinguished as the two main concepts [1]. Conventional classification approaches use pixels as smallest components of raster data. The digital numbers of these image elements can be used to group the image information into different semantic classes. Routinely, these approaches are widely applied to low and medium resolution remote sensing data. However, pixel-based classifiers hardly make use of adjacency and context information and thus are not suited to deal with the inherent heterogeneity within land-cover units. Furthermore, classification results usually suffer from a saltand-pepper effect and a post-processing, e.g. by filtering becomes necessary.

With the use of image segmentation techniques, some problems of pixel-based image analysis can be overcome. Segmentation means to partition an image into nonoverlapping homogeneous regions based on similarity criteria of gray values or textural properties [2] with the objective of generating segments which have a strong correlation with real objects of the Earth's surface. Due to the increasing spatial resolution of earth observation imagery per-parcel approaches gain in importance in the 
field of remote sensing image analysis. Especially for data of the one meter resolution SAR sensors (TerraSAR-X, COSMO-SkyMed) the use of segmentation-based methods appears promising. These images exhibit a very high spectral variance of the individual thematic classes due to the reduced mixed pixel phenomenon. In addition to spectral related characteristics of the image objects further parameters like contextual information, texture and object geometry can be used for an improved classification. A drawback of this technique is the high processing demand of the segmentation step, which mostly limits the size of the processed image in dependence on the used software package to less than 10,000 by 10,000 pixels [3]. This can be avoided by splitting the image into several parts and stitch them after the segmentation process.

Thresholding is one of the most frequently used techniques to distinguish water areas from land in SAR imagery (e.g. [4]). Thereby, all elements of the SAR intensity data with a radar cross-section lower than a given threshold are assigned to the water class. One of the main advantages of this method is its fast computational velocity. Additionally, the results are reliable and most of the extent of a smooth water surface can be derived by applying this technique. If available, ancillary information like digital elevation models can be used especially in significant topographic terrain to improve the mapping result.

On the one hand misclassified areas in higher regions than the main expanse of water, e.g. objects with a low radar cross section similar to calm water like streets, airstrips or radar shadow can be erased. On the other hand, topography information can be used in combination with contextual information to integrate water areas with backscatter intensities higher than the originally defined threshold, e.g. due to the effect of vegetation, into the water class. Thresholding works satisfactory for smooth water surfaces, which reflects radiation away from the SAR sensor, generating a very low signal return. In contrast, the surrounding terrain exhibits higher backscatter due to increased surface roughness. The applied threshold depends on the deviation of the water surface from a specular reflector due to influences of wind induced waves, precipitation, diffuse and corner reflecting vegetation as well as of the incidence angle of the sensor. Therefore, custom thresholds need to be set for every SAR scene individually.

Given this drawback different approaches have been developed for improved water classification techniques. These include e.g. fairly speckle and noise resistant active contour models (e.g. [5]), which had been used by e.g. [1], [6] and [7] for flood boundary delineation in medium resolution SAR imagery or fully automatic texture based maximum likelihood methods [7], which are able to detect water bodies independent of the sensor incidence angle.

In the context of flood mapping, multi-temporal analysis has proven superior to single data approaches. Different change detection approaches for the derivation of flood dynamics between SAR data have successfully been applied in the past. These include amplitude based (e.g. [1], [4]) as well as coherence based techniques (e.g. [8]). Using amplitude change detection in combination with C-Band multi-temporal SAR imagery flooded dense vegetation can be mapped e.g. by [9] due to the fact that microwaves at these wavelengths can penetrate the forest canopy. X-Band radiation with its minor wavelength however is volume scattered by the canopy and thus, no backscatter change is detectable between non-flooded and flooded vegetation. This makes the utilization of topographic information in combination with X-Band SAR data very important.

\subsection{Water body detection concept for TanDEM-X}

A key activity of the authors is to derive a water body dataset with a global coverage as a contribution to the TanDEM-X project. This upcoming German Aerospace Center (DLR) mission shall generate a worldwide, consistent, timely, high-precision Digital Elevation Model with an unprecedented accuracy corresponding to the HRTI-3 specifications (12 m posting, $2 \mathrm{~m}$ relative height accuracy for flat terrain) [10]. The planned launch of the TanDEM-X satellite will be by the end of 2009 .

A processing module is being developed, which shall detect water bodies reliably in TanDEM-X image data. Both amplitude and coherence values are used in this module. In a first step it will be tested, if the image area does contain potential water bodies. To realize this aim a global land/water mask that consists of the SRTM water mask and the GSHHS (Self-consistent, Hierarchical, High-resolution Shoreline) Database [11] will be used. Their water areas have to be expanded in order not to omit potential water bodies. Therefore a buffer distance will be applied to the water areas. This mask will be built up prior to the TanDEM-X data processing. It will be stored as a binary dataset in the framework of DLR's DEM database in a tile structure. The value of each tile indicates whether water bodies are expected or not. If a TanDEM-X raw data area contains only tiles with a dry area flag no water detection has to be done.

The primary aim of this dataset will be to exclude desert regions from the water mask generation which will save processing time. Furthermore the Polar Regions will be included in this dataset due to big variances in the water / ice boundary, which makes it impossible to generate a consistent water mask for these regions.

If the potential water body check is positive a set of analysis methods will be applied to the dataset. This will be a threshold analysis for the amplitude and coherence data. Further optional methods planned are the application of a dedicated texture filter and speckle analysis. These tools will be applied if the threshold analysis does not prove to be reliably enough for the detection of water bodies. The single 
result layers of the analysis tools are merged to one file and a classification is conducted. Shadow and layover data from a prior processing step of the raw data are used to exclude radar shadow areas in high mountainous terrain from the water mask. The result will be saved to a dataset which contains at least three probability levels that describe the probability of a single pixel of either being a water body or dry area.

According to the TanDEM-X data acquisition concept at least two datasets for every continental area on Earth will be recorded. The water body detection will be executed individually for the first year coverage and the second year coverage. After finishing of the TanDEM-X data acquisition all single water masks for a certain area are fused to finally classify the water area.

\subsection{Flood detection for Rapid Disaster Mapping}

In the event of a flood disaster, decision makers and relief organizations require a timely overview of the situation and detailed insights into the affected area in order to efficiently coordinate their emergency response measures. Since radar backscatter from water areas is influenced by numerous environmental and system-related factors (see chapter 2), the extraction of flood masks from X-Band SAR data is not trivial and requires a methodology that a) is robust and computationally efficient, b) is transferable to as many scenarios as possible and c) requires as little user-input as possible.

In the context of disaster mapping, a simple thresholding approach is probably most frequently used for separating grayscale values representing "water" and "land". A different approach currently applied during flood mapping activities at DLR's Center for Satellite Based Crisis Information (ZKI) is based on an image enhancement process which combines three logarithmized local image statistics (minimum / maximum, mean and variance). After the computation of these local statistics for a user-defined window size, the layers are employed in an unsupervised clustering algorithm. Water bodies are then interactively determined from the output cluster layer. In both approaches, a morphological plausibility check and refinement based on digital elevation models (DEM) can be applied to erase regions that are wrongly classified as water, e.g. forested areas in higher regions that are detected as flooded area because of their low backscatter. Depending on the degree of high-frequency spatial variance of the clustering, a post-classification clumping is used to remove single scatter pixels and achieve thematic layers with increased spatial coherency.

Along with recent developments in polarimetry, wavelength, spatial resolution and repetition rate given by new sensors such as TerraSAR-X, new approaches for the extraction of flood masks are currently being developed at DLR. The principal aim is to define methods which are as transferable as possible and only require little user interaction for the extraction of high resolution flood information.

\section{CONCLUSION}

This paper shows that the new SAR sensors like TerraSAR$\mathrm{X}$ have enormous potential for flood detection in disaster mapping activities due to their high geometric resolution and repetition rate. The thresholding approach works satisfactory only for smooth water surfaces. Due to the large amount of visible image objects and their spectral diversity the development of new segmentation-based and texturebased methods becomes necessary. To improve the classification results of the water detection the use of auxiliary data like DEM or GIS data is advisable.

\section{REFERENCES}

[1] Heremans R., Willekens A., Borghys D., Verbeeck B., Valckenborg J., Acheroy M., Perneel C., "Automatic detection of flooded areas on ENVISAT/ASAR images using an objectoriented classification technique and an active contour algorithm", Proceedings of IEEE Conference on RAST 2003, pp. 289-294, Istanbul, Turkey.

[2] Pal N.K., Pal S.K., "A review on image segmentation techniques”, Pattern Recognition, Vol. 26, pp. 1277-1294, 1993.

[3] Meinel G., Neubert M., “A comparison of segmentation programs for high resolution remote sensing data”, Proceedings of ISRPS 2004, Istanbul, Turkey.

[4] Townsend P.A., Walsh S.J., "Modelling flood plain inundation using integrated GIS with radar and optical remote sensing”, Geomorphology, Vol. 21(98), pp. 295-312, 1998.

[5] Horritt M.S., "A statistical active contour model for SAR image segmentation”, Image and Vision Computing, Vol. 17, pp. 213-224, 1999.

[6] Matgen P., Schumann G., Henry J.-B., Hoffmann L., Pfister L., "Integration of SAR-derived inundation areas, high precision topographic data and a river flow model toward real-time flood management", Journal of Applied Earth Observation and Geoinformation, Vol. 9, pp. 247-263, 2007.

[7] Ahtonen P., Euro M., Hallikainen M., Solbø S., Johansen B., Solheim I., "SAR and optical based algorithms for estimation of water bodies”, Technical report, FloodMan Project, http://projects.itek.norut.no/ floodman.

[8] Nico G., Pappalepore M., Pasquariello G., Refice A., Samarelli S., "Comparison of SAR amplitude vs. coherence flood detection methods - a GIS application”, International Journal of Remote Sensing, Vol. 21(8), pp. 1619-1631, 2000.

[9] Townsend P.A., "Relationships between forest structure and the detection of flood inundation in forest wetlands using C-band SAR”, Intern. Journ. of Remote Sensing, 23(3), pp. 443-460, 2002. [10] Zink M., Krieger G., Fiedler H., Hajnsek I., Moreira A., “The TanDEM-X Mission Concept”, Proceedings of EUSAR 2008, Vol. 4, pp. 31-34, Friedrichshafen, Germany.

[11] Wessel P., Smith W.H.F., "A Global, Self-consistent, Hierarchical, High-resolution Shoreline Database", Journal of Geophysical Research - Solid Earth, Vol. 101, Issue B4, pp. 87418743, 1996. 